\title{
Accurate free and forced rotational motions of rigid Venus
}

\author{
L. Cottereau, J. Souchay, and S. Aljbaae \\ Observatoire de Paris, Systèmes de Référence Temps Espace (SYRTE), CNRS/UMR8630, Paris, France \\ e-mail: Laure.cottereau@obspm.fr
}

Received 1 December 2009 / Accepted 27 February 2010

ABSTRACT

\begin{abstract}
Context. The precise and accurate modelling of a terrestrial planet like Venus is an exciting and challenging topic, all the more interesting because it can be compared with that of Earth for which such a modelling has already been achieved at the milli-arcsecond level.

Aims. We aim to complete a previous study, by determining the polhody at the milli-arcsecond level, i.e. the torque-free motion of the angular momentum axis of a rigid Venus in a body-fixed frame, as well as the nutation of its third axis of figure in space, which is fundamental from an observational point of view.

Methods. We use the same theoretical framework as Kinoshita (1977, Celest. Mech., 15, 277) did to determine the precession-nutation motion of a rigid Earth. It is based on a representation of the rotation of a rigid Venus, with the help of Andoyer variables and a set of canonical equations in Hamiltonian formalism.

Results. In a first part we computed the polhody, we showed that this motion is highly elliptical, with a very long period of $525 \mathrm{cy}$ compared with $430 \mathrm{~d}$ for the Earth. This is due to the very small dynamical flattening of Venus in comparison with our planet. In a second part we precisely computed the Oppolzer terms, which allow us to represent the motion in space of the third Venus figure axis with respect to the Venus angular momentum axis under the influence of the solar gravitational torque. We determined the corresponding tables of the nutation coefficients of the third figure axis both in longitude and in obliquity due to the Sun, which are of the same order of amplitude as for the Earth. We showed that the nutation coefficients for the third figure axis are significantly different from those of the angular momentum axis on the contrary of the Earth. Our analytical results have been validated by a numerical integration, which revealed the indirect planetary effects.
\end{abstract}

Key words. planets and satellites: individual: Venus - celestial mechanics

\section{Introduction}

Venus, which can be considered as the twin sister of the Earth with regard to its global characteristics (size, mass, density), has been the subject of a good amount of investigations on very long time scales to understand its slow retrograde rotation (243 d) and its rather small obliquity (2.63) (Goldstein 1964; Carpenter 1964; Goldreich \& Peale 1970; Lago \& Cazenave 1979; Dobrovoskis 1980; Yoder 1995; Correia \& Laskar 2001, 2003). Habibullin (1995) made an analytical study on the rotation of a rigid Venus. In Cottereau \& Souchay (2009) we presented an alternative study, from a theoretical framework already used by Kinoshita (1977) for the rigid Earth. We made an accurate description of the motion of rotation of Venus at short time scale. We calculated the ecliptic coordinates of the Venus orbital pole and the reference point $\gamma_{0 \mathrm{~V}}$, which is the equivalent of the vernal equinox for Venus. Our value for the precession in longitude was $\dot{\Psi}=4474$ ". $35 \mathrm{t} / \mathrm{cy} \pm 66.5$. We performed a full calculation of the nutation coefficients of Venus and presented the complete tables of nutation in longitude $\Delta \Psi$ and obliquity $\Delta \epsilon$ for the angular momentum axis due to both the dynamical flattening and triaxiality of the planet.

In this paper, the study begun in Cottereau \& Souchay (2009) is completed. First we consider in Sect. 2 the torque-free rotational motion of a rigid Venus. We recall the parametrization of Kinoshita (1977) and the important equations of Kinoshita (1972), which are used to solve this torque-free motion. The important characteristics (amplitude, period, trajectory) of the free motion are given. Cottereau \& Souchay (2009) assumed that the relative angular distances between the three poles (of angular momentum, figure and rotation) are very small as is the case for the Earth. In this paper we want to accurately determine the motion of the third Venus figure axis, which is the fundamental one from an observational point of view. To do that we reject the hypothesis of coincidence of the poles. Thus we determine the Oppolzer terms depending on the dynamical flattening and the trixiality of Venus. Then we compare these terms with the corresponding nutations terms for the angular momentum axis, as determined by Cottereau \& Souchay (2009) and the Oppolzer terms determined by Kinoshita (1977) for the Earth in Sect. 3. We give the complete tables of the nutation coefficients of the third figure axis of Venus. We compare them with the nutation coefficients of the angular momentum axis taken from Cottereau \& Souchay (2009) (Sect. 4). Finally in Sect. 5 we determine the nutation of the angular momentum axis by numerical integration using the ephemeris DE405. We validate the analytical results of Cottereau \& Souchay (2009) down to a precision of the order of a relative $10^{-5}$. We show that the discrepancies between the numerical integration and analytical results (Cottereau \& Souchay 2009) are caused by the indirect planetary effect, i.e. by the small contribution to the nutation, which is due to the periodic oscillations of the orbital motion of Venus. In this paper as in Cottereau $\&$ Souchay (2009) our domain of validity is roughly 3000 years.

\section{Torque-free motion for rigid Venus}

\subsection{Equation of torque-free motion}

We consider the problem of the rotational motion of the rigid Venus in absence of any external force. We note $(0, X, Y, Z)$ the 


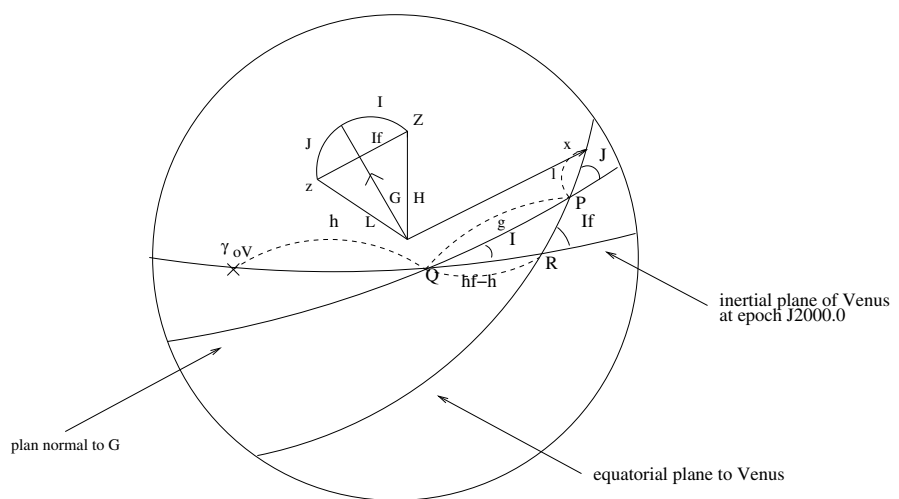

Fig. 1. Relation between the Euler angles and the Andoyer variables.

inertial frame and $(0, x, y, z)$ the cartesian coordinates fixed to the rigid body of the planet (see Fig. 1). The orientation of Venus with respect to the inertial axes is determined through the Euler angles $\left(h_{\mathrm{f}}, I_{\mathrm{f}}, \phi\right)$. The parameter $\phi$ gives the position of the prime meridian $(0, x)$ with respect to $\gamma_{0 V}$ (Kinoshita 1977). The angular momentum axis (hereafter denoted AMA) of Venus is the axis directed along $G$. The components of the angular momentum referred to the body-fixed axes are:

$L_{x}=A \omega_{x}=A\left(\dot{h}_{\mathrm{f}} \sin I_{\mathrm{f}} \sin \phi+\dot{I}_{\mathrm{f}} \cos \phi\right)$

$L_{y}=B \omega_{y}=B\left(\dot{h}_{\mathrm{f}} \sin I_{\mathrm{f}} \cos \phi-\dot{I}_{\mathrm{f}} \sin \phi\right)$

$L_{z}=C \omega_{z}=C\left(\dot{h_{\mathrm{f}}} \cos I_{\mathrm{f}}+\dot{\phi}\right)$,

where $A, B, C$ are the principal inertia moments of Venus. Moreover the kinetic energy is

$T=\frac{1}{2}\left(\omega_{x} L_{x}+\omega_{y} L_{y}+\omega_{z} L_{z}\right)$.

To describe the torque-free rotational motion we use the Andoyer variables (Andoyer 1923; Kinoshita 1972) (see Fig. 1):

- $L$ the angular momentum component along the $0 z$ axis;

- $H$ the angular momentum component along the $0 Z$ axis;

- $G$ the angular momentum amplitude of Venus;

- $l$ the angle between the origin meridian $O x$ and the node $P$;

- $h$ the longitude of the node of the AMA with respect to $\gamma_{0 V}$;

- $g$ the longitude of the plane node $(0, X, Y)$ with respect to $Q$ and to the equatorial plane.

This parametrization is described in detail in Kinoshita (1972, 1977) and in Cottereau \& Souchay (2009). From these definitions we have

$L=G \cos J, \quad H=G \cos I$,

where $I, J$ are the angle between the AMA and the inertial axis $(O, Z)$ and the angle between the AMA and the third figure axis (hereafter denoted TFA) respectively. Using spherical trigonometry, we determine the following relation between the variables:

$\phi=l+g$.

The components of the angular momentum vector with the Andoyer variables are

$L_{x}=\sqrt{G^{2}-L^{2}} \sin l$

$L_{y}=\sqrt{G^{2}-L^{2}} \cos l$

$L_{z}=L$.

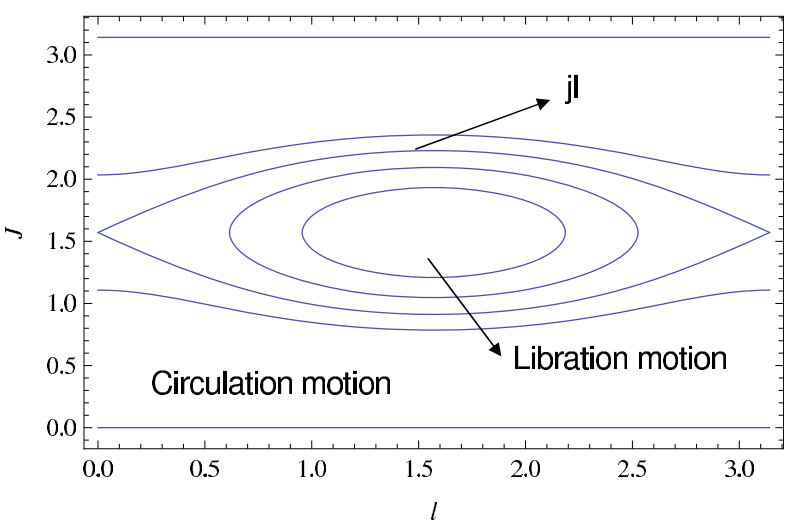

Fig. 2. Isoenergetic curves of Venus in the $(J, l)$ phase space of the torque-free motion. Two motions are showen: "the libration motion" and the "circulation motion".

The Hamiltonien for the torque-free motion of Venus corresponding to the kinetic energy is

$H=\frac{1}{2}\left(\frac{\sin ^{2} l}{A}+\frac{\cos ^{2} l}{B}\right)\left(G^{2}-L^{2}\right)+\frac{L^{2}}{2 C}$.

The Hamiltonian $H$ does not depend on time and is free from $g$ and $h$. Thus the number of degrees of freedom of the torque-free motion is one. Deprit (1967) has characterized this motion by studying the isoenergetic curves in the phase plane $L-l$. But we prefer to study the isoenergetic curves in the $(J, l)$ phase space using Eq. (3). This allows a good description of the position of the AMA with respect to the TFA. Figure 2 shows the two possible motions: one is the libration motion and the other one the circulation motion. Using the values of the relative differences of the moments of inertia of Williams (private communication)

$$
\begin{aligned}
& \frac{C-A}{M R^{2}}=5.519 \times 10^{-6}, \frac{C-B}{M R^{2}}=3.290 \times 10^{-6}, \\
& \frac{B-A}{M R^{2}}=2.228 \times 10^{-6}
\end{aligned}
$$

and addopting $\frac{C}{M R^{2}}=0.3360$ (Yoder 1995), we get $\frac{C}{A}=$ $1.000016, \frac{C}{B}=1.000010$. We introduce here $j$, which corresponds to the minimum value of $J$ when $l=\frac{\pi}{2}$. We denote by $j_{l}$, the value reached by $j$ on the separatrix. The libration motion is not possible if $J<j_{l}$ or $J>\left(\pi-j_{l}\right)$. The numerical values of $j_{l}$ can be determined with the equations of Kinoshita (1972). We have

$j_{l}=\arccos \sqrt{\frac{2 e}{1+e}}$,

with

$e=\frac{1}{2}\left(\frac{1}{B}-\frac{1}{A}\right) D$

and

$\frac{1}{D}=\frac{1}{C}-\frac{1}{2}\left(\frac{1}{B}+\frac{1}{A}\right)$

where $e$ measures the triaxiality of the rigid body (Andoyer 1923). Applying these formulas to Venus we get $j_{l}=52^{\circ} .23$. 


\subsection{Canonical transformations}

To determine the torque-free motion of Venus, we use the method described in full detail by Kinoshita (1972). We give here only the important results and equations which are needed to apply this study to Venus. The Hamiltonian for the torquefree motion is given by Eq. (6). To solve the equations of motion, we perform a canonical transformation which replaces the angular momentum by its action variable (Goldstein 1964). First we make two intermediate transformations to simplify the computation.

\subsubsection{First transformation}

We change $(L, G, l, g)$ to $\left(\alpha_{1}, \alpha_{2}, u_{1}, u_{2}\right)$ using the Hamilton Jacobi method (Chazy 1953). We note $S\left(L, G, \alpha_{1}, \alpha_{2}\right)$ the characteristic function. We have the Hamilton-Jacobi equation

$F_{0}=\frac{1}{2}\left(\frac{\sin ^{2} l}{A}+\frac{\cos ^{2} l}{B}\right)\left(\left(\frac{\partial S}{\partial g}\right)^{2}-\left(\frac{\partial S}{\partial l}\right)^{2}\right)+\frac{1}{2 C}\left(\frac{\partial S}{\partial l}\right)^{2}$,

where

$\alpha_{1}=F_{\mathrm{o}}, \alpha_{2}=\frac{\partial S}{\partial g}=G$.

This equation can be solved as

$S=\int \sqrt{\frac{\gamma^{2}-e \alpha_{2}^{2} \cos 2 l}{1-e \cos 2 l}} \mathrm{~d} l+\alpha_{2} g$,

where

$\gamma^{2}=\left[2 \alpha_{1}-\frac{1}{2}\left(\frac{1}{A}+\frac{1}{B}\right) \alpha_{2}^{2}\right] D$.

The constants $e$ and $D$ are given by Eqs. (9) and (10). Because the new Hamiltonian depends on only one of the momenta $\alpha_{1}$, we have

$u_{1}=t+\beta_{1}=\frac{\partial S}{\partial \alpha_{1}}=\frac{\partial S}{\partial \gamma} \frac{D}{\gamma}$

$u_{2}=\beta_{2}=\frac{\partial S}{\partial \alpha_{2}}$.

\subsubsection{Second transformation}

Then we make another transformation, which changes $\left(\alpha_{1}, \alpha_{2}\right.$, $\left.u_{1}, u_{2}\right)$ to $(\bar{L}, \bar{G}, \bar{l}, \bar{g})$. The two momenta are defined as follows:

$\bar{L}=\gamma$

$\bar{G}=\alpha_{2}=G$.

With these new coordinates the Hamiltonian $F_{0}$ becomes

$F_{0}=\frac{1}{2 D}{\overline{L_{1}}}^{2}+\frac{1}{4}\left(\frac{1}{A}+\frac{1}{B}\right){\overline{G_{1}}}^{2}$.

Thanks to the Hamilton equations the conjugate variables are

$\bar{l}=\frac{\partial F_{0}}{\partial \bar{L}}=\frac{\bar{L}}{D}\left(t+\beta_{1}\right)$

$\bar{g}=\frac{\partial F_{0}}{\partial \bar{G}}=\frac{1}{2}\left(\frac{1}{A}+\frac{1}{B}\right) \bar{G}(t+\beta 1)+\beta_{2}$.

Using (16) we get $\bar{J}$ from

$\bar{L}=\bar{G} \cos \bar{J}$ and

$\cos \bar{J}=\sqrt{1-(1+e) \sin ^{2} j}=\cos j \sqrt{1-e \tan ^{2} j}$,

where $j$ is defined in Sect. 2.1. Now we can introduce the canonical transformation, which replaces the angular momentum by its action variable.

\subsubsection{Action variables}

The action variables are given by:

$\tilde{L}=\frac{1}{2 \pi} \oint \sqrt{\frac{\bar{L}^{2}-e \bar{G}^{2} \cos 2 l}{1-e \cos 2 l}} \mathrm{~d} l$

$\tilde{G}=\bar{G}=G=\alpha_{2}$,

where $\tilde{l}$ and $\tilde{g}$ are canonically conjugate variables to $\tilde{L}$ and $\tilde{G}$ respectively. We make the following transformation to simplify the calculation:

$\cos 2 \delta=\frac{\cos 2 l-e}{1-e \cos 2 l} \quad$ or $\quad \cos 2 l=\frac{\cos 2 \delta+e}{1+e \cos 2 \delta}$.

We obtain

$\tilde{L}=\frac{\bar{G}}{2 \pi} \frac{\sqrt{1-e^{2}}}{\sqrt{\bar{b}}} \oint \frac{\sqrt{1-\bar{k}^{2} \cos ^{2} \delta}}{1+e \cos 2 \delta} \mathrm{d} \delta=\bar{G} \wedge_{0}$,

where

$\bar{k}^{2}=\frac{2 e}{1-e}(\bar{b}-1) \quad \bar{b}=\left(\cos ^{2} j\right)^{-1}$

and

$\wedge_{0}=\frac{2}{\pi}\left[E(k) F\left(\chi, k^{\prime}\right)+K(k) E\left(\chi, k^{\prime}\right)-K(k) F\left(\chi, k^{\prime}\right)\right]$,

with

$\chi=\sin ^{-1} \sqrt{\frac{1}{\bar{b}}} \quad k^{\prime}=\sqrt{1-\bar{k}^{2}}$.

In Eq. (26) $K(k)$ is the complete elliptic integral of the first kind with modulus $\bar{k}^{2}, E(k)$ that of the second kind. $F\left(\chi, k^{\prime}\right)$ is an incomplete elliptic integral of the first kind, $E\left(\chi, k^{\prime}\right)$ that of the second kind, $\wedge_{0}$ is a Heumann lambda function (Byrd \& Friedman 1954). Because the complete solution of Eq. (24) is of no interest here, we only give the mean motion of the angular variables $\tilde{l}$ and $\tilde{g}$ that will be used bellow (Kinoshita 1972, 1992). With the canonical transformations the time variation of $\tilde{l}$ and $\tilde{g}$ can be determined. We have

$\tilde{g}=\tilde{n_{g}} t+\beta_{2}=\tilde{n_{g}} t+\tilde{g_{0}}$

$\tilde{l}=\tilde{n}_{l} t$

where

$\tilde{n}_{l}=\frac{\pi \bar{G}}{2 K D} \sqrt{1-e^{2}} \cos j$

$\tilde{n_{g}}=\frac{G}{C}-\tilde{n_{l}} \wedge_{0}-G\left(\frac{1}{C}-\frac{1}{A}\right) \sin ^{2} j$

Here the epoch of time $t$ is defined so that $\tilde{l}=\frac{\pi}{2}$ at $t=0$. Now we can give the development of the variables $g$ and $l$, which describe the torque-free motion. 
Table 1. Important values of the free motion of Venus.

\begin{tabular}{ll}
\hline \hline Constant & Numerical values \\
\hline$\frac{C}{A}$ & 1.000016 \\
$\frac{C}{B}$ & 1.000010 \\
$e$ & 0.230769 \\
$\frac{C}{D}$ & -0.000013 \\
$n_{l}$ & $0.0119495 \mathrm{rd} / \mathrm{cy}$ \\
$T_{l}$ & $525.81 \mathrm{cy}$ \\
$n_{g}$ & $0.0258549 \mathrm{rd} / \mathrm{d}$ \\
$T_{g}$ & $-243.02 \mathrm{~d}$ \\
\hline
\end{tabular}

\subsection{Venus free rotation}

As a result we can use a development of our variables $g, l$ and $J$ with respect to $j$ and $e$. The results of these technical developments (Kinoshita 1972) are summarized in the Appendix. As is the case for the Earth we assume that the angle $j$ of Venus is very small. Table 1 gives the numerical values of the important constants used in the theory of the free motion of Venus. To calculate these values we take $\frac{C}{M R^{2}}$ (Yoder 1995) and the values of the moment of inertia given in Eq. (7). The value of $D$ and $\tilde{b}$ are unknown. Finally we find

$$
\begin{aligned}
l= & l^{*}-0.0576923 j^{2} \frac{\sin 2 \tilde{l}}{1+e \cos 2 \tilde{l}}+O\left(j^{4}\right) \\
g= & +\frac{G}{D n_{l}}\left[-0.973009\left(l^{*}-\tilde{l}\right)\right. \\
& +0.307692 \times j^{2}\left[0.230769 \frac{\sin 2 \tilde{l}}{1+e \cos 2 \tilde{l}}\right. \\
& \left.+2.05548\left(l^{*}-\tilde{l}\right)\right]+O\left(j^{4}\right) \\
J & =j \sqrt{1.28713+0.287129 \cos 2 \tilde{l}}+O\left(e^{3}, j^{2}\right) \\
\tan l^{*} & =0.797007 \tan \tilde{l} .
\end{aligned}
$$

Using the developments for a small $e$, we get

$$
\begin{aligned}
l= & \tilde{l}-0.111538 \sin 2 \tilde{l}+0.006221 \sin 4 \tilde{l}+O\left(e^{3}, j^{2}\right) \\
g= & \tilde{g}+0.111538 \sin 2 \tilde{l}-0.006221 \sin 4 \tilde{l}+O\left(e^{3}, j^{2}\right) \\
J= & \tilde{J}[1.00933+0.11154 \cos 2 \tilde{l}]+O\left(e^{3}, j^{2}\right) \\
\tilde{J}= & j+0.115385 \tan j \\
& +0.05325 \tan j\left(\frac{1}{8}+\frac{3}{16} \tan ^{2} j\right)+O\left(e^{3}\right) .
\end{aligned}
$$

Because the main limitation of our calculation is the uncertainty on the ratio $\frac{C}{M R^{2}}$ of Venus (Yoder 1995), our polynomial expansions must be done accordingly. Fourth order terms are too small compared to our level of accuracy and have been discarded. Projecting the pole of Venus on the $(X, Y)$ plane with a value of $j=0.01 \mathrm{rd}$ for the developments above, we can plot the free motion of Venus (Fig. 2).

We note here that the value of $j$ has been chosen arbitrarily, for it does not significantly affect the polhody, except the amplitude. We see that the torque-free motion of Venus is an elliptic motion, as is the case for the Earth. The rotational free motion of Venus, with a period $T_{l}=525$ centuries (Table 1 ) is

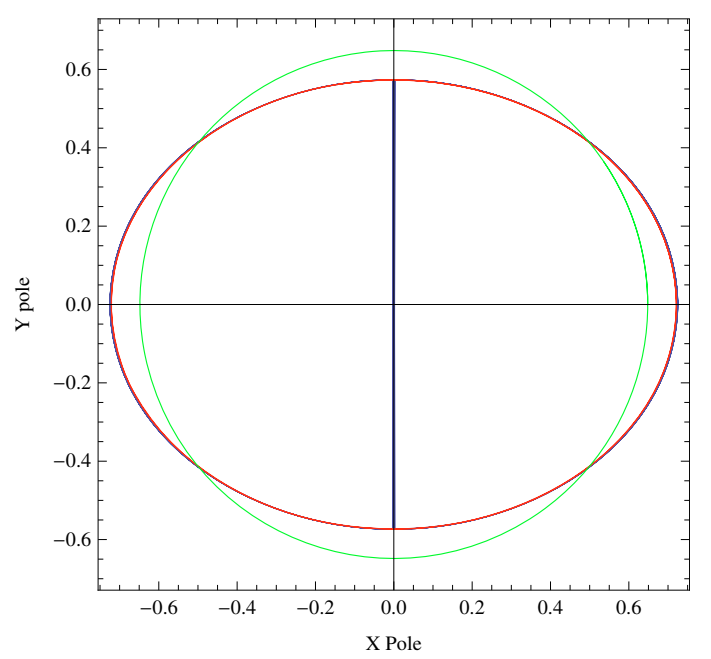

Fig. 3. $(X, Y)$ free motion of Venus in space for five hundred century time space (red and blue curves). The green curve represents a circle with the radius $r=\bar{j}$. We take $j=0.01 \mathrm{rd}$.

much slower than that of Earth (303 d). If we consider the elastic Earth (ocean and atmosphere), the torque-free motion has a period of $432 \mathrm{~d}$, significantly larger than in the rigid case. The atmosphere of Venus is much denser than that of the Earth. So it would be interesting to study the torque-free motion of the elastic Venus in a next paper.

\section{Rigid Venus forced rotational motion}

In Cottereau \& Souchay (2009) we assumed that the relative angular distances between the three poles of Venus (pole of angular momentum, of figure and of rotation) are very small, as is the case for the Earth. In this section we determine the motion of the TFA, which is the fundamental one from an observational point of view. To do so we reject the hypothesis of coincidence of the poles. Using the spherical trigonometry in the triangle $(P, Q, R)$ (see Fig. 1) we determine the relations between the TFA and the AMA. Supposing that the angle $J$ between the AMA and the TFA is small we obtain

$$
\begin{aligned}
& h_{\mathrm{f}}=h+\frac{J}{\sin I} \sin g+O\left(J^{2}\right) \\
& I_{\mathrm{f}}=I+J \cos g+O\left(J^{2}\right),
\end{aligned}
$$

where $I$ characterizes the obliquity and $h$ the motion of precession-nutation in longitude of the AMA of Venus. $h_{\mathrm{f}}$ and $I_{\mathrm{f}}$ correspond to the same definitions as $h$ and $I$, but for the TFA instead of the AMA. This yields (Kinoshita 1977)

$$
\begin{aligned}
& \Delta h_{\mathrm{f}}=\Delta h+\Delta\left(\frac{J \sin g}{\sin I}\right)+O\left(J^{2}\right) \\
& \Delta I_{\mathrm{f}}=\Delta I+\Delta(J \cos g)+O\left(J^{2}\right),
\end{aligned}
$$

where $\Delta h$ and $\Delta I$ represent the variation of the nutation of Venus in longitude and obliquity respectively. The second term on the right hand side of Eqs. (35) and (36) are the so-called Oppolzer terms. They represent the difference between the TFA nutation and the AMA nutation (in longitude and in obliquity). These terms represent the differential effects of solid body tides on both 
axes. Developing Eqs. (35) and (36) we obtain

$\Delta\left(\frac{J \sin g}{\sin I}\right)=\frac{1}{\sin I}\left(\Delta J \sin g+J \Delta g \cos g-\frac{J \sin g \Delta I \cos I}{\sin ^{2} I}\right)$

$\Delta(J \cos g)=\Delta J \cos g-J \sin g \Delta g$.

We see that Eqs. (37) and (38) are functions of $\Delta g$ and $\Delta J$. We recall here that $g$ and $J$ caracterize the motion of the AMA with respect to the TFA. As the Earth has a fast rotation, the TFA and the AMA can be considered to be identical. Venus has a slow rotation, so it is interesting to see what the difference will be with respect to the TFA motion. To determine $\Delta g$ and $\Delta J$ we must solve the equations of motion.

\subsection{Equations of motion}

The Hamiltonian related to the rotational motion of Venus is (Cottereau \& Souchay 2009)

$K^{\prime \prime}=F_{\mathrm{o}}+E+E^{\prime}+U$,

where $F_{\mathrm{o}}$ is the Hamiltonian for the free motion, $E+E^{\prime}$ is a component related to the motion of the orbit of Venus, which is caused by planetary perturbations. The expression of $F_{\mathrm{o}}$ has been set in the previous section. The expression of $E+E^{\prime}$ is given in detail in Cottereau \& Souchay (2009). $U$ is the disturbing potential due to the external disturbing body considered. Here the sole external disturbing body is the Sun (the perturbation due to the planets can be neglected in first order), and its disturbing potential is given by

$U=\frac{G M^{\prime}}{r^{3}}\left[\frac{2 C-A-B}{2} P_{2}(\sin \delta)+\frac{A-B}{4} P_{2}^{2}(\sin \delta) \cos 2 \alpha\right]$,

where $\boldsymbol{G}$ is the gravitationnal constant, $M^{\prime}$ is the mass of the Sun, $r$ is the distance between the barycenters of the Sun and Venus. $\alpha$ and $\delta$ are the planetocentric longitude and latitude of the Sun respectively, with respect to the mean equator of Venus and with respect to a meridian of origin (therefore $\alpha$ must not be confused with the usual right ascension). The $P_{n}^{m}$ are the classical Legendre functions given by

$P_{n}^{m}(x)=\frac{(-1)^{m}\left(1-x^{2}\right)^{\frac{m}{2}}}{2^{n} n !} \frac{d^{n+m}\left(x^{2}-1\right)^{n}}{d^{n+m} x}$.

In Eq.(40) we only consider the potential in first order. The method for solving the equation of motion is described in Kinoshita (1977). Only the final results are given. We have

$\Delta g=\frac{1}{G}\left(\cot J \frac{\partial W_{1}}{\partial J}+\cot I \frac{\partial W_{1}}{\partial I}\right)$

$\Delta J=\frac{1}{G}\left(\frac{1}{\sin J} \frac{\partial W_{1}}{\partial l}-\cot J \frac{\partial W_{1}}{\partial g}\right)$

where

$$
\begin{aligned}
W_{1}= & \int \frac{\boldsymbol{G} M^{\prime}}{r^{3}}\left[\frac{2 C-A-B}{2} P_{2}(\sin \delta)\right. \\
& \left.+\frac{A-B}{4} P_{2}^{2} \sin \delta \cos 2 \alpha\right] \mathrm{d} t .
\end{aligned}
$$

We use a transformation described by Kinoshita (1977) and based on the Jacobi polynomials. It expresses $\alpha$ and $\delta$ as functions of $\lambda$ and $\beta$, respectively the longitude and the latitude of the
Sun with respect to the Venus mean orbital plane

$$
\begin{aligned}
P_{2}(\sin \delta)= & \frac{1}{2}\left(3 \cos ^{2} J-1\right)\left[\frac{1}{2}\left(3 \cos ^{2} I-1\right) P_{2}(\sin \beta)\right. \\
& \left.-\frac{1}{2} \sin 2 I P_{2}^{1}(\sin \beta) \cos 2(\lambda-h)\right] \\
& +\sin 2 J\left[-\frac{3}{4} \sin 2 I P_{2}(\sin \beta) \cos g\right. \\
& -\sum_{\epsilon= \pm 1} \frac{1}{4}(1+\epsilon \cos I)(-1+2 \epsilon \cos I) \\
& \times P_{2}^{1}(\sin \beta) \sin (\lambda-h-\epsilon g) \\
& -\sum_{\epsilon= \pm 1} \frac{1}{8} \epsilon \sin I(1+\epsilon \cos I) \\
& \left.\times P_{2}^{2}(\sin \beta) \cos (2 \lambda-2 h-\epsilon g)\right]+\sin ^{2} J \\
& \times\left[\frac{3}{4} \sin { }^{2} I P_{2}(\sin \beta) \cos 2 g+\frac{1}{4} \sum_{\epsilon= \pm 1} \epsilon \sin I\right. \\
& \times(1+\epsilon \cos I) P_{2}^{1}(\sin \beta) \sin (\lambda-h-2 \epsilon g)-\frac{1}{16} \\
& \left.\times \sum_{\epsilon= \pm 1}(1+\epsilon \cos I)^{2} P_{2}^{2}(\sin \beta) \cos 2(\lambda-h-\epsilon g)\right] .
\end{aligned}
$$

and

$$
\begin{aligned}
P_{2}^{2}(\sin \delta) \cos 2 \alpha= & 3 \sin ^{2} J\left[-\frac{1}{2}\left(3 \cos ^{2} I-1\right) P_{2}(\sin \beta)\right. \\
\times & \cos 2 l+\frac{1}{4} \sum_{\epsilon= \pm 1} \sin 2 I P_{2}^{1}(\sin \beta) \sin (\lambda-h-2 \epsilon l) \\
& \left.+\frac{1}{8} \sin ^{2} I P_{2}^{2}(\sin \beta) \cos 2(\lambda-h-\epsilon l)\right] \\
& +\sum_{\rho= \pm 1} \rho \sin J(1+\rho \cos J) \\
& \times\left[-\frac{3}{2} \sin 2 I P_{2}(\sin \beta) \cos (2 \rho l+g)\right. \\
& -\sum_{\epsilon= \pm 1} \frac{1}{2}(1+\epsilon \cos I)(-1+2 \epsilon \cos I) \\
& \times P_{2}^{1}(\sin \beta) \sin (\lambda-h-2 \rho \epsilon l-\epsilon g) \\
& -\sum_{\epsilon= \pm 1} \frac{1}{4} \epsilon \sin I(1+\epsilon \cos I) \\
& \left.\times P_{2}^{2}(\sin \beta) \cos (2 \lambda-2 h-2 \rho \epsilon l-\epsilon g)\right]
\end{aligned}
$$

To simplify the calculations, we separately study the symmetric part of Eq. (44), which depends on the dynamical flattening, and the antisymmetric part, which depends on the triaxiality of Venus. So the dynamical flattening coefficient of will be noted with an " $s$ " index and the triaxiality coefficient with an " $a$ " index. From its definition above, we can set $\beta \approx 0$, because the latitude of the Sun with respect to the Venus mean orbital plane can be considered as null. 


\subsection{Oppolzer terms depending on the dynamical flattening}

Using Eqs. (44) and (45) with $\beta=0$, we have

$$
\begin{aligned}
W_{s 1}= & \frac{\boldsymbol{G} M^{\prime}}{a^{3}} \frac{2 C-A-B}{2} \int\left[\left(\frac{a}{r}\right)^{3} P_{2}(\sin \delta)\right] \mathrm{d} t \\
= & \frac{1}{2}\left(3 \cos ^{2} J-1\right) W_{s 10}-\frac{1}{2} \sin 2 J W_{s 11} \\
& +\frac{1}{4} \sin ^{2} J W_{s 12},
\end{aligned}
$$

where

$$
\begin{aligned}
W_{s 10}= & K_{s^{\prime}}\left[-\frac{1}{6}\left(3 \cos ^{2} I-1\right) \int \frac{1}{2}\left(\frac{a}{r}\right)^{3} \mathrm{~d} t\right. \\
& \left.-\frac{1}{4} \sin ^{2} I \int \cos 2(\lambda-h)\left(\frac{a}{r}\right)^{3} \mathrm{~d} t\right] \\
W_{s 11}= & K_{s^{\prime}}\left[-\frac{1}{2} \sin 2 I \int \frac{1}{2}\left(\frac{a}{r}\right)^{3} \cos g \mathrm{~d} t\right. \\
& -\frac{1}{4}(1-\cos I) \int \cos (2 \lambda-2 h+g)\left(\frac{a}{r}\right)^{3} \mathrm{~d} t \\
& \left.+\frac{1}{4}(1+\cos I) \int \cos (2 \lambda-2 h-g)\left(\frac{a}{r}\right)^{3} \mathrm{~d} t\right] \\
W_{s 12}= & K_{s^{\prime}}\left[-\sin ^{2} I \frac{1}{2} \int\left(\frac{a}{r}\right)^{3} \cos 2 g \mathrm{~d} t\right. \\
& -\frac{1}{4}(1-\cos I)^{2} \int \cos 2(\lambda-h+g)\left(\frac{a}{r}\right)^{3} \mathrm{~d} t \\
& \left.-\frac{1}{4}(1+\cos I)^{2} \int \cos 2(\lambda-h-g)\left(\frac{a}{r}\right)^{3} \mathrm{~d} t\right],
\end{aligned}
$$

and

$K_{s^{\prime}}=\frac{3 G M^{\prime}}{a^{3}} \frac{2 C-A-B}{2}$.

According to the Hamilton equations, we obtain

$$
\begin{aligned}
\Delta_{\mathrm{s}} g= & \frac{1}{G}\left[-3 \cos ^{2} J W_{s 10}-\frac{\cos 2 J \cos J}{\sin J} W_{s 11}\right. \\
& \left.+\frac{1}{2} \cos ^{2} J W_{s 12}\right] \\
& -\cos I \Delta h+O\left(J^{2}\right) .
\end{aligned}
$$

We assume that the angle $J$ is small as for the Earth. This yields

$$
\begin{aligned}
\Delta_{s} g= & \frac{1}{G}\left[-3 W_{s 0}-\frac{1}{J} W_{s 11}+\frac{1}{2} W_{s 12}\right] \\
& -\cos I \Delta h+O\left(J^{2}\right) .
\end{aligned}
$$

We have also

$$
\begin{aligned}
\Delta_{\mathrm{s}} J & =\frac{1}{G}\left[\cos ^{2} J \frac{\partial W_{s 11}}{\partial g}-\frac{1}{8} \sin 2 J \frac{\partial W_{s 12}}{\partial g}\right] \\
& =\frac{1}{G} \frac{\partial W_{s 11}}{\partial g}+O(J) .
\end{aligned}
$$

Table 2. Development of $\frac{1}{2}\left(\frac{a}{r}\right)^{3} \cos (g)$ of Venus. $t$ is counted in Julian centuries.

\begin{tabular}{rrrrrr}
\hline \hline$M$ & $L_{\mathrm{S}}$ & $g$ & $\begin{array}{r}\text { Period } \\
\mathrm{d}\end{array}$ & $\cos \times 10^{-7}$ & $t \cos \times 10^{-7}$ \\
\hline 0 & 0 & 1 & -243.02 & $\left(\frac{1}{2}+\frac{3}{4} e^{2}\right)=5000344$ & -48 \\
1 & 0 & 1 & 2980.71 & $\left(\frac{3}{4} e+\frac{27}{32} e^{3}\right)=50792$ & -3582 \\
1 & 0 & -1 & 116.75 & $\left(\frac{3}{4} e+\frac{27}{32} e^{3}\right)=50792$ & -3582 \\
2 & 0 & 1 & 208.948 & $\left(\frac{9}{8} e^{2}\right)=516$ & -72 \\
2 & 0 & -1 & 76.83 & $\left(\frac{9}{8} e^{2}\right)=516$ & -72 \\
3 & 0 & 1 & 108.27 & $\frac{53}{16} e^{3}=10$ & 0 \\
3 & 0 & -1 & 57.25 & $\frac{53}{16} e^{3}=10$ & 0 \\
\hline
\end{tabular}

Table 3. Development of $\left(\frac{a}{r}\right)^{3} \cos (2(\lambda-h)-g)$ of Venus. $t$ is counted in Julian centuries.

\begin{tabular}{lcccrr}
\hline \hline$M$ & $L_{\mathrm{S}}$ & $g$ & $\begin{array}{c}\text { Period } \\
\mathrm{d}\end{array}$ & $\cos \times 10^{-7}$ & $t \cos \times 10^{-7}$ \\
\hline 0 & 2 & -1 & 76.83 & $\left(1-\frac{5}{2} e^{2}\right)=9998853$ & 161 \\
-1 & 2 & -1 & 116.75 & $\left(-\frac{1}{2} e+\frac{1}{16} e^{3}\right)=-33859$ & 2388 \\
1 & 2 & -1 & 57.25 & $\left(\frac{7}{2} e-\frac{123}{16} e^{3}\right)=236993$ & -16718 \\
2 & 2 & -1 & 45.62 & $\left(\frac{17}{2} e^{2}\right)=3898$ & -550 \\
-3 & 2 & -1 & -2980.71 & $\frac{1}{48} e^{3}=0$ & 0 \\
3 & 2 & -1 & 37.92 & $\frac{845}{48} e^{3}=54$ & -4 \\
\hline
\end{tabular}

Table 4. Development of $\left(\frac{a}{r}\right)^{3} \cos (2(\lambda-h)+g)$ of Venus. $t$ is counted in Julian centuries.

\begin{tabular}{lcccrr}
\hline \hline$M$ & $L_{\mathrm{S}}$ & $g$ & $\begin{array}{c}\text { Period } \\
\mathrm{d}\end{array}$ & $\cos \times 10^{-7}$ & $t \cos \times 10^{-7}$ \\
\hline 0 & 2 & 1 & 208.95 & $\left(1-\frac{5}{2} e_{V}^{2}\right)=9998853$ & 161 \\
-1 & 2 & 1 & 2980.71 & $\left(-\frac{1}{2} e+\frac{1}{16} e^{3}\right)=-33859$ & 2388 \\
1 & 2 & 1 & 108.27 & $\left(\frac{7}{2} e-\frac{123}{16} e^{3}\right)=236993$ & -16718 \\
2 & 2 & 1 & 73.06 & $\left(\frac{17}{2} e^{2}\right)=3898$ & 550 \\
-3 & 2 & 1 & -116.75 & $\frac{1}{48} e^{3}=0$ & 0 \\
3 & 2 & 1 & 55.14 & $\frac{845}{48} e^{3}=54$ & -4 \\
\hline
\end{tabular}

Using Eqs. (52) and (53) we obtain the Oppolzer terms depending on the dynamical flattening

$\Delta_{\mathrm{s}}\left(\frac{J \sin g}{\sin I}\right)=\frac{1}{G \sin I}\left[\frac{\partial W_{s 11}}{\partial g} \sin g-W_{s 11} \cos g\right]+O(J)$

$\Delta_{\mathrm{S}}(J \cos g)=\Delta(J) \cos g-J \Delta g \sin g$

$$
=\frac{1}{G}\left[\frac{\partial W_{s 11}}{\partial g} \cos g+W_{s 11} \sin g\right]+O(J)
$$

To solve Eqs. (54) and (55) through $W_{s 11}$ given by Eq. (48), it is necessary to develop $\frac{1}{2}\left(\frac{a}{r}\right)^{3} \cos g,\left(\frac{a}{r}\right)^{3} \cos (2 \lambda-2 h+g)$ and $\left(\frac{a}{r}\right)^{3} \cos (2 \lambda-2 h-g)$ with respect to the mean anomaly $M$, the mean longitude of the Sun $L_{S}$ and $g$, the angle determined in Sect. 2. With Kepler's law we obtain this development (see Tables 2-4). Our value of the eccentricity was taken from Simon et al. (1994).

The numerical value for $T_{g}$ is given in Table 1 . We recall here that our domain of validity is 3000 years as it was in Cottereau \& Souchay (2009). 
L. Cottereau et al.: Accurate free and forced rotational motions of rigid Venus

Table 5. Oppolzer terms in longitude depending on dynamical flattening $\left[\Delta \Psi_{\mathrm{s}}=\Delta h_{\mathrm{s}}\right.$ : nutation coefficients of the AMA $]$.

\begin{tabular}{crrrr}
\hline \hline Argument & $\begin{array}{r}\text { Period } \\
\mathrm{d}\end{array}$ & $\begin{array}{r}\sin \omega t \\
\operatorname{arcsec} \\
\left(10^{-} 7\right)\end{array}$ & $\begin{array}{r}t \sin \omega t \\
\text { arcsec/julian century } \\
\left(10^{-} 7\right)\end{array}$ & $\begin{array}{r}\cos \omega t \\
\operatorname{arcsec} \\
\left(10^{-} 7\right)\end{array}$ \\
\hline $2 L_{\mathrm{s}}$ & 112.35 & $14962988[-21900468]$ & $-242[-352]$ & $0[0]$ \\
$M$ & 224.70 & $-6134235[889997]$ & $432651[-62765]$ & $5416[-61]$ \\
$2 L_{\mathrm{s}}+M$ & 74.90 & $264403[-346057]$ & $-18651[24412]$ & $-5[-7]$ \\
$2 L_{\mathrm{s}}-M$ & 224.70 & $-76066[148323]$ & $5366[-10461]$ & $2[-10]$ \\
$2 L_{\mathrm{s}}+2 M$ & 56.17 & $3466[-4269]$ & $-489[602]$ & $0[0]$ \\
$2 M$ & 112.35 & $-5749[4521]$ & $811[-640]$ & $0[0]$ \\
\hline
\end{tabular}

Table 6. Oppolzer terms in the obliquity depending on dynamical flattening $\left[\Delta \epsilon_{\mathrm{s}}=\Delta I_{\mathrm{s}}\right.$ : nutation coefficients of the AMA $]$.

\begin{tabular}{crrrr}
\hline \hline Argument & $\begin{array}{r}\text { Period } \\
\mathrm{d}\end{array}$ & $\begin{array}{r}\text { cos } \omega t \\
\operatorname{arcsec} \\
\left(10^{-} 7\right)\end{array}$ & $\begin{array}{r}t \cos \omega t \\
\operatorname{arcsec} / \text { Julian century } \\
\left(10^{-} 7\right)\end{array}$ & $\begin{array}{r}\sin \omega t \\
\operatorname{arcsec} \\
\left(10^{-} 7\right)\end{array}$ \\
\hline $2 L_{\mathrm{s}}$ & 112.35 & $-690090[100741]$ & $-11[16]$ & $0[0]$ \\
$M$ & 224.70 & $-260831[0]$ & $1840[0]$ & $-248[0]$ \\
$2 L_{\mathrm{s}}+M$ & 74.90 & $-12184[15919]$ & $859[-1123]$ & $0[0]$ \\
$2 L_{\mathrm{s}}-M$ & 224.70 & $3594[-6822]$ & $-253[481]$ & $0[0]$ \\
$2 L_{\mathrm{s}}+2 M$ & 56.17 & $-160[196]$ & $22[-27]$ & $0[0]$ \\
$2 M$ & 112.35 & $-122[0]$ & $17[0]$ & $0[0]$ \\
\hline
\end{tabular}

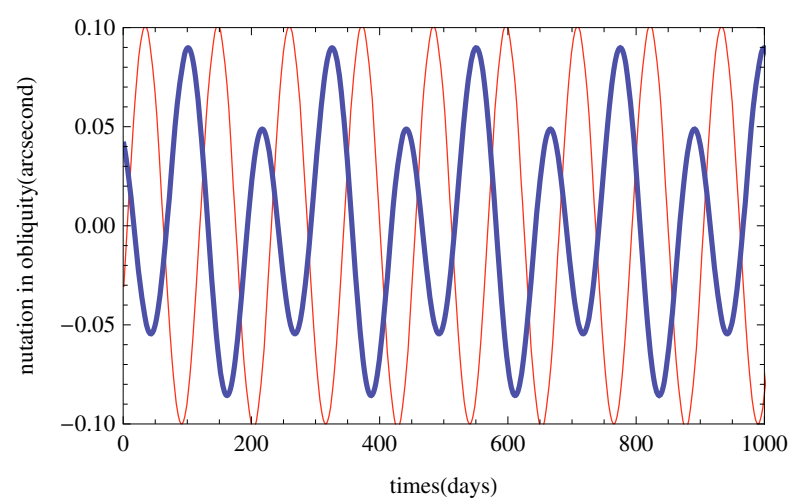

Fig. 4. Nutation of the AMA (red curve) and nutation of the Oppolzer terms (blue and bolt curve) in the obliquity of Venus depending on its dynamical flattening for a $1000 \mathrm{~d}$ span, from J2000.0.

Because of the the developments above we give in Tables 5 and 6 the Oppolzer terms, in longitude and in obliquity respectively, depending on the dynamical flattening. For comparison we give the corresponding nutation coefficients (in brackets) of the AMA determined in Cottereau \& Souchay (2009). They are represented in both Figs. 4 and 5 for a $1000 \mathrm{~d}$ time span to see the leading oscillations. We remark that the Oppolzer terms are of the same order of magnitude as the nutation coefficients. The Oppolzer terms associated with the argument $M$ are even larger. This is due to the low value of $\dot{M}+\dot{g}=-2 \Pi /(-243.02 / 36525)+$ $2 \Pi /(224.70 / 36525)=76.99 \mathrm{rd} / \mathrm{cy}$, which enters in the denominator during the integration of the equations of motion. Whereas for the calculation of the corresponding AMA coefficient only the numerical value $\dot{M}=2 \Pi /(224.70 / 36525)=1021.33 \mathrm{rd} / \mathrm{cy}$ appears, which is higher than $\dot{M}+\dot{g}$. In Figs. 4 and 5, showing the Oppolzer terms, the presence of the sinusoid with a period of $224 \mathrm{~d}$ reflects this. Remark (Table 6) that the Oppolzer terms associated with the argument $M$ and $2 M$ have a non zero amplitude, whereas the nutation coefficients of the AMA associated

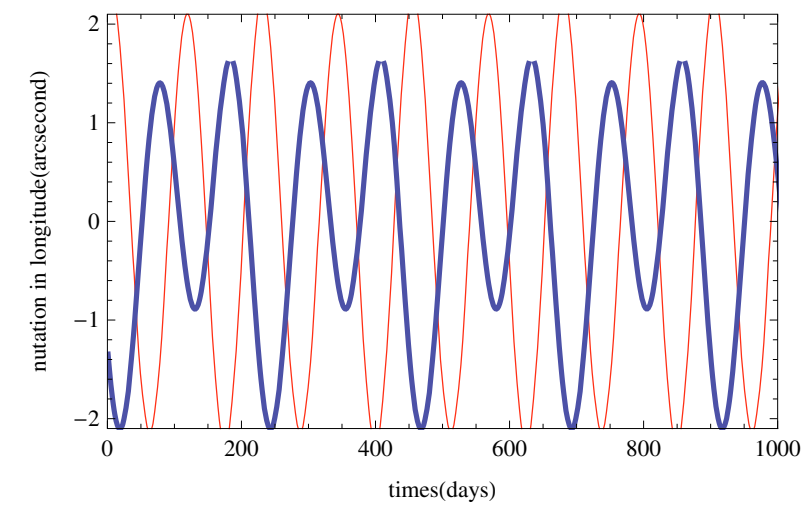

Fig. 5. Nutation of the AMA (red curve) and nutation of the Oppolzer terms (blue and bolt curve) in longitude of Venus depending on its dynamical flattening for a $1000 \mathrm{~d}$ span, from J2000.0.

with the same arguments do not exist. Indeed we remark here that to compute the nutation coefficients in obliquity for this axis, we performed the derivative of $W_{1}$ with respect to $h$, which does not appear in the terms associated with the argument $M$ and $2 M$. Notice also that for the Earth (see Kinoshita 1977), the Oppolzer terms depending on the dynamical flattening are negligible with respect to the nutation coefficients of the AMA. The largest Oppolzer term in longitude in Kinoshita (1977) is 0.'007559, whereas for Venus it is 1'.4962. In obliquity it is $0 .{ }^{\prime} 002762$, whereas for Venus it is $0 .{ }^{\prime} 6901$. The rapid rotation of the Earth compared with the slow retrograde rotation of Venus explains this contrast: the frequencies, which depend on the rotation $g$ and enter in the denominator during the integration, are $10^{4}$ times higher for the Earth than for Venus.

Due to its slow rotation, the triaxiality of Venus $\left(1.66 \times 10^{-6}\right)$ is not negligible compared to the dynamical flattening $\left(1.31 \times 10^{-5}\right)$. Thus the Oppolzer terms depending on the triaxiality must be considered and are calculated below. 


\subsection{Oppolzer terms depending on the triaxiality}

From Eq. (44), we have

$$
\begin{aligned}
W_{a 1}= & \frac{G M^{\prime}}{a^{3}} \frac{A-B}{4} \int\left[\left(\frac{a}{r}\right)^{3} P_{2}^{2}(\sin \delta) \cos 2 \alpha\right] \mathrm{d} t \\
= & \frac{3}{2} \sin ^{2} J W_{a 10}-\sum_{\rho= \pm 1} \rho \sin J(1+\rho \cos J) W_{a 11(\rho)} \\
& +\sum_{\rho= \pm 1} \frac{1}{4}(1+\cos J)^{2} W_{a 12(\rho)},
\end{aligned}
$$

where

$$
\begin{aligned}
W_{a 10}= & K_{a^{\prime}}\left[\left(3 \cos ^{2} I-1\right) \int \frac{1}{2}\left(\frac{a}{r}\right)^{3} \cos 2 l \mathrm{~d} t\right. \\
+ & \frac{1}{4} \sin ^{2} I \int\left(\frac{a}{r}\right)^{3} \cos 2(\lambda-h+l) \mathrm{d} t \\
+ & \frac{1}{4} \sin ^{2} I \int\left(\frac{a}{r}\right)^{3} \cos 2(\lambda-h-l) \mathrm{d} t \\
W_{a 11(\rho)}= & K_{a^{\prime}}\left[-\frac{1}{2} \sin 2 I \int\left(\frac{1}{2} \frac{a}{r}\right)^{3} \cos (2 \rho l+g) \mathrm{d} t\right. \\
& -\frac{1}{4} \sin I(1-\cos I) \\
& \times \int\left(\frac{a}{r}\right)^{3} \cos (2 \lambda-2 h+2 \rho l+g) \mathrm{d} t \\
& +\frac{1}{4} \sin I(1+\cos I) \\
& \left.\times \int\left(\frac{a}{r}\right)^{3} \cos (2 \lambda-2 h-2 \rho l-g) \mathrm{d} t\right] \\
& +\frac{1}{4}(1+\cos I)^{2} \\
& \left.+\frac{1}{4}(1-\cos I)^{2} \int\left(\frac{a}{r}\right)^{3} \cos 2(\lambda-h-\rho l-g) \mathrm{d} t\right], \\
W_{a 12(\rho)}= & K_{a^{\prime}}\left[\sin ^{2} I \int\left(\frac{1}{2} \frac{a}{r}\right)^{3} \cos (2 l+2 \rho g) \mathrm{d} t\right. \\
& \\
&
\end{aligned}
$$

\begin{tabular}{|c|c|c|c|}
\hline Argument & $\begin{array}{r}\text { Period } \\
\mathrm{d}\end{array}$ & $\begin{array}{r}\text { Oppolzer } \\
\sin (\omega t) \\
\operatorname{arcsec} \\
10^{-} 7\end{array}$ & $\begin{array}{r}\text { A.M } \\
\text { LC } \sin (\omega t) \\
\operatorname{arcsec} \\
10^{-} 7\end{array}$ \\
\hline $2 \Phi$ & -121.51 & -11967515 & 5994459 \\
\hline $2 L_{\mathrm{S}}-2 \Phi$ & 58.37 & -3784751 & 2880826 \\
\hline$M+2 \Phi$ & -264.6 & 1490497 & 132590 \\
\hline$M-2 \Phi+2 L_{\mathrm{S}}$ & 46.34 & -66849 & 54201 \\
\hline$M-2 \Phi$ & 78.86 & 58400 & -39519 \\
\hline $2 L_{\mathrm{S}}+2 \Phi$ & 1490.35 & 5448 & -38866 \\
\hline$-M-2 \Phi+2 L_{\mathrm{S}}$ & 78.86 & 19476 & -13179 \\
\hline $2 M+2 \Phi$ & 1490.35 & 1062 & -7587 \\
\hline $2 M-2 \Phi+2 L_{\mathrm{S}}$ & 38.41 & -876 & 739 \\
\hline $2 M-2 \Phi$ & 58.37 & 390 & -297 \\
\hline$M+2 \Phi+2 L_{\mathrm{S}}$ & 195.26 & 67 & 121 \\
\hline$-M+2 \Phi+2 L_{\mathrm{S}}$ & -264.66 & -263 & -23 \\
\hline $2 M+2 \Phi+2 L_{\mathrm{S}}$ & 104.47 & 1 & -1 \\
\hline
\end{tabular}

and

$$
K_{a^{\prime}}=\frac{3 G M^{\prime}}{a^{3}} \frac{A-B}{4} \text {. }
$$

Using the Hamilton equations and assuming, as for the terms depending on the dynamical flattening, that $J$ is a small angle, we obtain

$$
\begin{aligned}
\Delta_{A g}= & \frac{1}{G}\left[3 W_{a 10}-W_{a 12(\rho)}-2 J W_{a 11(1)}\right] \\
& -\cos I \Delta_{A h}+O\left(J^{2}\right),
\end{aligned}
$$

and

$$
\begin{aligned}
\Delta_{a J}= & \frac{1}{G \sin J} \frac{\partial W_{a 1}}{\partial l}-\frac{1}{G} \cot J \frac{\partial W_{a 1}}{\partial g}=\frac{3}{2} \sin J \frac{\partial W_{a 10}}{\partial l} \\
& -\frac{1+\cos J}{G}\left[\frac{\partial W_{a 11(1)}}{\partial l}-\cos J \frac{\partial W_{a 11(1)}}{\partial g}\right] \\
& +\frac{(1+\cos J)^{2}}{4 G \sin J}\left[\frac{\partial W_{a 12(\rho)}}{\partial l}-\cos J \frac{\partial W_{a 12(\rho)}}{\partial g}\right] .
\end{aligned}
$$

Table 7. Oppolzer terms in longitude depending on triaxiality.

Notes. Comparison with the corresponding nutation coefficients of the AMA in the tables of Cottereau \& Souchay (2009).

As $W_{a 11(-1)}$ is multiplied by $(1-\cos J)$, with our hypothesis it disappears from Eqs. (61) and (62). Since $W_{a 11(1)}$ and $W_{a 12(\rho)}$ include $l$ and $g$ in the form of $g+2 l$ and $2 g+2 l$ respectively, the last terms in Eqs. (62) are negligible. Therefore $\Delta_{a J}$ becomes

$\Delta_{a J}=-\frac{2}{G} \frac{\partial W_{a 11(1)}}{\partial g}+O(J)$

The Oppolzer terms depending on the triaxiality are

$$
\begin{aligned}
\Delta\left(\frac{J \sin g}{\sin I}\right)= & -\frac{2}{G \sin I}\left[\frac{\partial W_{a 11(1)}}{\partial g} \sin g\right. \\
& \left.+W_{a 11(1)} \cos g\right]+O(J)
\end{aligned}
$$

$$
\begin{aligned}
\Delta(J \cos g) & =\Delta J \cos g-J \Delta g \sin g \\
& =-\frac{2}{G}\left[\frac{\partial W_{a 11(1)}}{\partial g} \cos g-W_{a 11(1)} \sin g\right] .
\end{aligned}
$$

To solve Eqs. (64) and (65) it is necessary to develop $\frac{1}{2}\left(\frac{a}{r}\right)^{3} \cos (2 l+g),\left(\frac{a}{r}\right)^{3} \cos (2 \lambda-2 h+2 l+g)$ and $\left(\frac{a}{r}\right)^{3} \cos (2 \lambda-2 h-$ $2 l-g$ ) with respect to the mean anomaly $M$, the mean longitude of the Sun $L_{\mathrm{s}}$ and the angles $l$ and $g$ determined in Sect. 2.3. The coefficients are the same as those in Tables 3 and 4. Only the corresponding periods are different, because their calculation includes the argument $l$. The period $T_{l}$ is very long, as shown in Table 1. Considering our level of accuracy, we suppose in this section that $e$ is constant and we take the value of Simon et al. (1994) as $e=0.006771$.

We can determine the Oppolzer terms depending on the triaxiality.

Tables 7 and 8 give the Oppolzer terms in longitude and in obliquity respectively, depending on Venus triaxiality. For comparison, we give the corresponding nutation coefficients of the AMA determined in Cottereau \& Souchay (2009). They are represented in both Figs. 6 and 7 for a $4000 \mathrm{~d}$ time span. We remark here also that the Oppolzer terms are more important than the corresponding nutation coefficients of the AMA. The Oppolzer terms associated with the argument $2 \Phi$ is even roughly twice larger than the corresponding nutation coefficient. This is due to the low value 
Table 8. Oppolzer terms in obliquity depending on triaxiality.

\begin{tabular}{|c|c|c|c|}
\hline Argument & $\begin{array}{r}\text { Period } \\
\mathrm{d}\end{array}$ & $\begin{array}{r}\text { Oppolzer } \\
\cos (\omega t) \\
\operatorname{arcsec} \\
10^{-} 7\end{array}$ & $\begin{array}{r}\text { A.M } \\
\text { CS } \cos (\omega t) \\
\operatorname{arcsec} \\
10^{-} 7\end{array}$ \\
\hline $2 \Phi$ & -121.51 & 555905 & -275453 \\
\hline $2 l_{\mathrm{S}}-2 \Phi$ & 58.37 & -173668 & 132365 \\
\hline$M+2 \Phi$ & -264.6 & -68544 & -6093 \\
\hline$M-2 \Phi+2 L_{\mathrm{S}}$ & 46.34 & 3074 & 2491 \\
\hline $2 l_{\mathrm{S}}+2 \Phi$ & 1490.35 & -250 & 1786 \\
\hline$M-2 \Phi$ & 78.86 & 2686 & 1816 \\
\hline$-M-2 \Phi+2 L_{\mathrm{S}}$ & 78.86 & 896 & -606 \\
\hline $2 M+2 \Phi$ & 1490.35 & -49 & 348 \\
\hline $2 M-2 \Phi+2 L_{\mathrm{S}}$ & 38.41 & -40 & 35 \\
\hline $2 M-2 \Phi$ & 58.37 & 18 & 14 \\
\hline$M+2 \Phi+2 L_{S}$ & 195.26 & -3 & 6 \\
\hline$-M+2 \Phi+2 L_{S}$ & -264.6 & 12 & 1 \\
\hline $2 M+2 \Phi+2 L_{\mathrm{S}}$ & 104.47 & 0 & 0 \\
\hline
\end{tabular}

Notes. Comparison with the corresponding nutation coefficients of the AMA in the tables of Cottereau \& Souchay (2009).

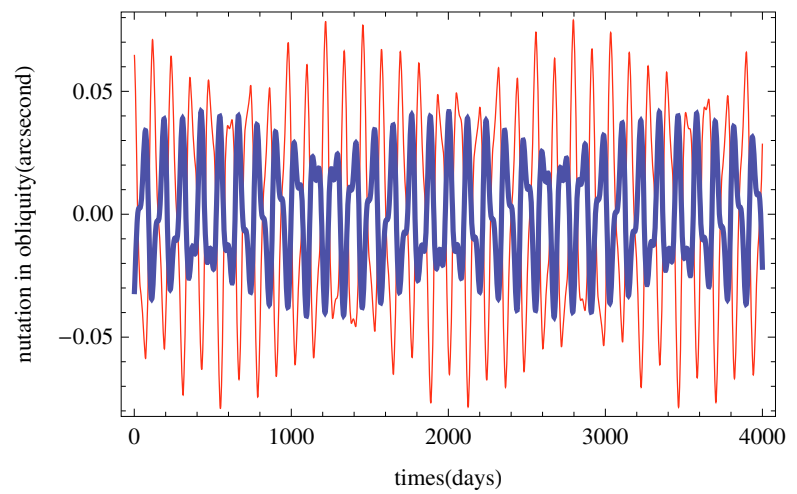

Fig. 6. Nutation of the AMA (Blue and bolt curve) and nutation of the Oppolzer terms (red curve) in obliquity of Venus depending on its triaxility for a $4000 \mathrm{~d}$ span, from $\mathrm{J} 2000.0$.

of $\dot{2 l}+\dot{g}=2 \Pi / 525.81-2 \Pi /(243.02 / 36525)=944.36 \mathrm{rd} / \mathrm{cy}$, which appears in the denominator during the integration of the equations of motion, whereas for the calculation of the corresponding coefficient of the AMA only the sidereal angle frequency $\dot{2 \Phi} \approx \dot{\varphi} l+\dot{2} g=1888.68 \mathrm{rd} / \mathrm{cy}$ appears which, is significantly larger. The appearance of the angle $\dot{g}$ during the integration explains that the other Oppolzer terms are more important than the corresponding coefficient of the nutation of the AMA, as justified in Sect. 3.2 for the terms depending on the dynamical flattening.

Other than for Earth, the Oppolzer terms in triaxiality are not negligible compared to the nominal values of the corresponding nutation coefficient. The frequencies depending on the rotation $2 l+g$, which enter in the denominator during the integration, are very small compared those of our planet. Now we can determine the nutation of the TFA of Venus, which is fundamental from an observational point of view.

\section{Numerical results and comparison with the motion of the AMA}

From Eqs. (35) and (36) we calculate the nutation coefficients of the TFA. We recall here that the nutation is respectively designated in longitude by $\Delta h_{\mathrm{f}}$ and in obliquity by $\Delta I_{\mathrm{f}}$. Tables 9

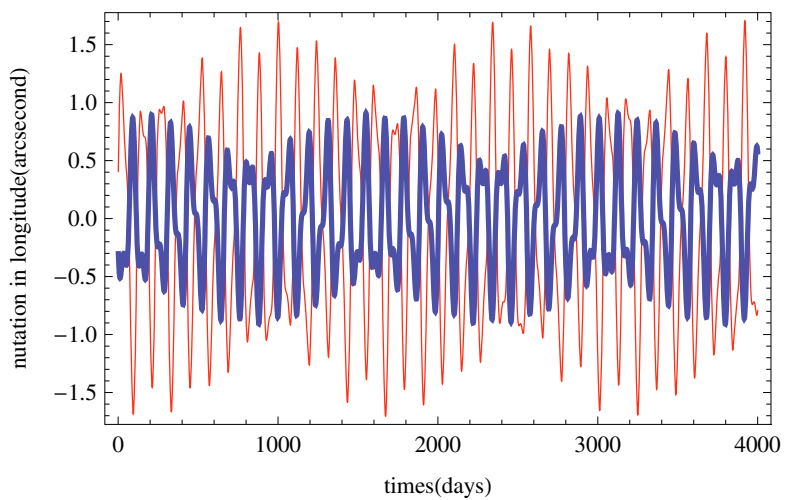

Fig. 7. Nutation of the AMA (Blue and bolt curve) and nutation of the Oppolzer terms (red curve) in longitude of Venus depending on its triaxility for a $4000 \mathrm{~d}$ span, from $\mathrm{J} 2000.0$.

Table 9. $\Delta \Psi_{\mathrm{fs}}=\Delta h_{\mathrm{fs}}$ : nutation coefficients of the TFA in longitude of Venus depending on its dynamical flattening.

\begin{tabular}{crrrr}
\hline \hline Argument Period & $\begin{array}{c}\sin \omega t \\
\operatorname{arcsec} \\
\left(10^{-} 7\right)\end{array}$ & $\begin{array}{r}t \sin \omega t \\
\text { arcsec/julian century } \\
\left(10^{-} 7\right)\end{array}$ & $\begin{array}{r}\cos \omega t \\
\left(10^{-} 7\right)\end{array}$ \\
\hline $2 L_{\mathrm{s}}$ & 112.35 & -6937480 & -594 & 0 \\
$M$ & 224.70 & -5244238 & 369886 & 5355 \\
$2 L_{\mathrm{s}}+M$ & 74.90 & -81654 & 5760 & -2 \\
$2 L_{\mathrm{s}}-M$ & 224.70 & 72257 & -5095 & -8 \\
$2 L_{\mathrm{s}}+2 M$ & 56.17 & -803 & 113 & $0[0]$ \\
$2 M$ & 112.35 & -1228 & 171 & $0[0]$ \\
\hline
\end{tabular}

Table 10. $\Delta \epsilon_{\mathrm{fs}}=\Delta I_{\mathrm{fs}}$ : nutation coefficients of the TFA in obliquity of Venus depending on its dynamical flattening.

\begin{tabular}{crrrr}
\hline \hline Argument Period & $\begin{array}{l}\text { cos } \omega t \\
\text { arcsec } \\
\left(10^{-} 7\right)\end{array}$ & $\begin{array}{r}t \cos \omega t \\
\operatorname{arcsec} / \text { Julian } \\
\text { century } \\
\left(10^{-} 7\right)\end{array}$ & $\begin{array}{r}\sin \omega t \\
\operatorname{arcsec} \\
\left(10^{-} 7\right)\end{array}$ \\
\hline $2 L_{\mathrm{s}}$ & 112.35 & -589348 & 5 & 0 \\
$M$ & 224.70 & -260831 & 1839 & -248 \\
$2 L_{\mathrm{s}}+M$ & 74.90 & 3735 & -264 & 0 \\
$2 L_{\mathrm{s}}-M$ & 224.70 & -3228 & -228 & 0 \\
$2 L_{\mathrm{s}}+2 M$ & 56.17 & 37 & -4 & 0 \\
$2 M$ & 112.35 & -122 & 17 & 0 \\
\hline
\end{tabular}

and 10 give the nutation coefficients depending on the dynamical flattening in longitude and in obliquity respectively. In a similar way, Tables 11 and 12 give the coefficients depending on the triaxiality.

In this section we will show the difference between the nutation of the TFA, calculated in this paper and that of the AMA of Venus ( $\delta I$ and $\Delta h)$, as calculated by Cottereau \& Souchay (2009). Figures 8 and 9 represent the nutation in longitude and in obliquity of the two axes respectively for a $4000 \mathrm{~d}$ time span.

Concerning the longitude we can point out two important specific remarks:

- The nutation of the TFA is significantly smaller than the nutation of the AMA. Indeed the amplitude peak to peak of the nutation of the TFA (an amplitude of 1.5') is twice as small as that of the AMA (an amplitude of $3^{\prime \prime}$ ).

- The nutation of the TFA is dominated by three sinusoids associated with the arguments $2 L_{\mathrm{s}}, M$ and $2 \Phi$, with respective periods $112.35 \mathrm{~d}, 224.70 \mathrm{~d}$ and $121.51 \mathrm{~d}$ whereas the nutation 
Table 11. $\Delta \Psi_{\mathrm{fa}}=\Delta h_{\mathrm{fa}}$ nutation coefficients of the TFA in longitude of Venus depending on its triaxiality.

\begin{tabular}{crr}
\hline \hline Argument & $\begin{array}{r}\text { Period } \\
\mathrm{d}\end{array}$ & $\begin{array}{r}\text { sin }(\omega t) \\
\operatorname{arcsec} \\
10^{-} 7\end{array}$ \\
\hline $2 \Phi$ & -121.51 & -5973056 \\
$2 L_{\mathrm{S}}-2 \Phi$ & 58.37 & -903925 \\
$M+2 \Phi$ & -264.6 & 1623087 \\
$M-2 \Phi+2 L_{\mathrm{S}}$ & 46.34 & -12648 \\
$M-2 \Phi$ & 78.86 & 29199 \\
$2 L_{\mathrm{S}}+2 \Phi$ & 1490.35 & -33418 \\
$-M-2 \Phi+2 L_{\mathrm{S}}$ & 78.86 & 6297 \\
$2 M-2 \Phi+2 L_{\mathrm{S}}$ & 38.41 & $-137]$ \\
$2 M-2 \Phi$ & 58.37 & 195 \\
$M+2 \Phi+2 L_{\mathrm{S}}$ & 195.26 & 188 \\
$-M+2 \Phi+2 L_{\mathrm{S}}$ & -264.66 & -286 \\
$2 M+2 \Phi+2 L_{\mathrm{S}}$ & 104.47 & 0 \\
\hline
\end{tabular}

Table 12. $\Delta \epsilon_{\mathrm{fa}}=\Delta I_{\mathrm{fa}}$ : nutation coefficients of the TFA in obliquity of Venus depending on its triaxiality.

\begin{tabular}{crr}
\hline \hline Argument & $\begin{array}{r}\text { Period } \\
\mathrm{d}\end{array}$ & $\begin{array}{r}\cos (\omega t) \\
\operatorname{arcsec}\end{array}$ \\
\hline $2 \Phi$ & -121.51 & 280452 \\
$2 l_{\mathrm{S}}-2 \Phi$ & 58.37 & -41303 \\
$M+2 \Phi$ & -264.6 & -74637 \\
$M-2 \Phi+2 L_{\mathrm{S}}$ & 46.34 & 5565 \\
$2 l_{\mathrm{S}}+2 \Phi$ & 1490.35 & 1536 \\
$M-2 \Phi$ & 78.86 & 4502 \\
$M-2 \Phi+2 L_{\mathrm{S}}$ & 78.86 & 290 \\
$2 M+2 \Phi$ & 1490.35 & 299 \\
$2 M-2 \Phi+2 L_{\mathrm{S}}$ & 38.41 & -5 \\
$2 M-2 \Phi$ & 58.37 & 32 \\
$M+2 \Phi+2 L_{\mathrm{S}}$ & 195.26 & 3 \\
$-M+2 \Phi+2 L_{\mathrm{S}}$ & -264.6 & 13 \\
$2 M+2 \Phi+2 L_{\mathrm{S}}$ & 104.47 & 0 \\
\hline
\end{tabular}

of the AMA is dominated by two sinusoids of argument $2 L_{\mathrm{s}}$ and $2 \Phi$.

The same kind of remark is available in obliquity, but the difference between the nutations is less important. The amplitude of the nutation of the TFA is varying between 0 . $^{\prime} 10$ and $0 .^{\prime} 08$ (peak to peak), whereas the AMA amplitude is varying between -0 .' $^{\prime} 14$ and 0 ' $^{\prime} 11$. The nutation in obliquity is also dominated by a sinusiod, with a period $M$ which is not as large as the corresponding AMA sinusoid.

Finally we can highlight the differences between the Earth and Venus. For the Earth, the nutation of the two axes (angular momentum and third figure axis) are roughly the same (Woolard 1953; Kinoshita 1977), whereas for Venus they are significantly different. We can also remark that the leading nutation component of the third Venus figure axis in longitude due to the gravitational action of the Sun, with argument $2 L_{\mathrm{S}}$ (see Eq. (9)) has an amplitude of $0{ }^{\prime} 693$. This is of the same order as the leading $2 L_{\mathrm{s}}$ the nutation amplitude of the Earth due to the Sun, i.e. 0 O' $^{\prime} 998$ despite the fact that Venus has a very small dynamical flattening. As explained by Cottereau \& Souchay (2009) this is due to the compensating role of the very slow rotation of Venus. Moreover, notice that for Venus the argument $L_{\mathrm{S}}$ stands for the longitude of the Sun as seen from the planet, so that the corresponding period of the leading nutation term with $2 L_{\mathrm{s}}$ argument is $112.35 \mathrm{~d}$, whereas it is $182.5 \mathrm{~d}$ for the Earth.

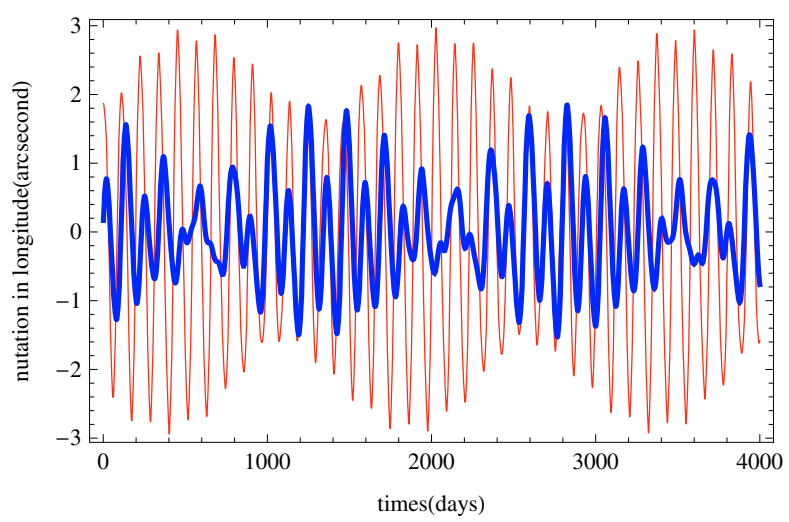

Fig. 8. Nutation of the TFA (blue curve) and nutation of the momentum axis in longitude of Venus for $4000 \mathrm{~d}$ time span, from J2000.0.

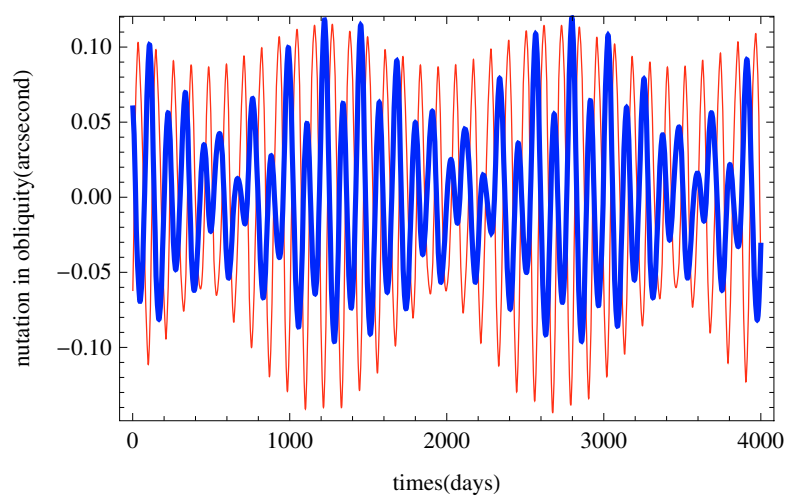

Fig. 9. Nutation of the TFA (blue curve) and nutation of the momentum axis in obliquity of Venus for $4000 \mathrm{~d}$ time span, from J2000.0.

\section{Determination of the indirect planetary effects on the nutation of Venus}

Using the ephemeris DE405 (Standish 1998), we computed the nutation of Venus by numerical integration with a Runge-Kutta 12th order algorithm. Figures 10 and 11 show the differences between the nutations in obliquity and in longitude of the AMA as computed from the analytical tables (Cottereau \& Souchay 2009) and that from the numerical integration for a $4000 \mathrm{~d}$ time span. The residuals obtained clearly consist of periodic components with small amplitudes of the order of $10^{-5 \prime \prime}$ in obliquity and $10^{-3 \prime \prime}$ in longitude. This numerical integration validates the results of Cottereau \& Souchay (2009) down to a relative accuracy of $10^{-5}$. Moreover Kinoshita's model used in Cottereau \& Souchay (2009) assumed a Keplerian motion of Venus around the Sun. It is well known that the effects of planetary attraction into Earth's orbit (called indirect planetary effect) entails a departure from the Keplerian motion and that this departure induces new nutation terms as calculated by Souchay \& Kinoshita (1996). In order to infer whether the discrepancies between our numerical integration and our analytical computation are caused by this indirect planetary effect in Venus orbit, we performed a spectral analysis of the residuals.

The leading oscillations of the two signals (in longitude and in obliquity) are determined with a fast Fourier Transform (FFT). Tables 13 and 14 give the leading amplitudes and periods of the sinusoids characterizing the signal in Figs. 10 and 11, where the curve at the bottom represents the residuals after subtraction of these sinusoids. The periods presented in the Tables 13 and 14 do not correspond to any period of the tables given in the precedent section starting from the keplerian approximation. On 


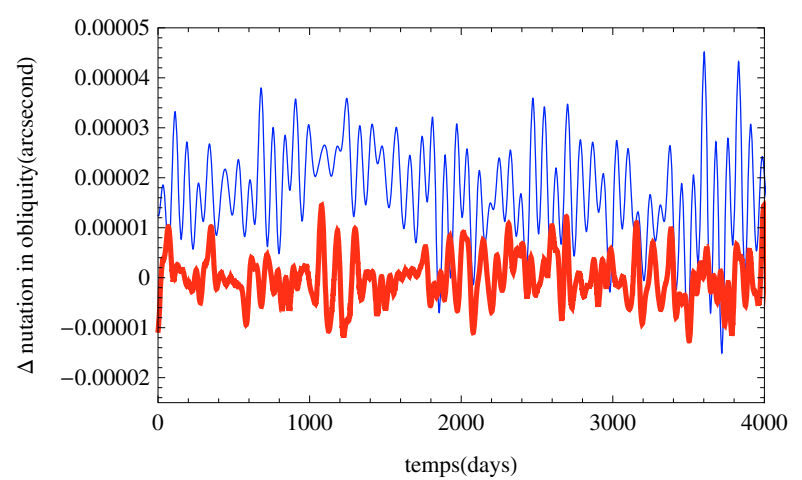

Fig. 10. Difference between the obliquity nutation of the AMA and the numerical integration for $4000 \mathrm{~d}$ time span, from J2000.0. The curve at bottom represents the residual after substracting the sinusoidal terms of Table 13

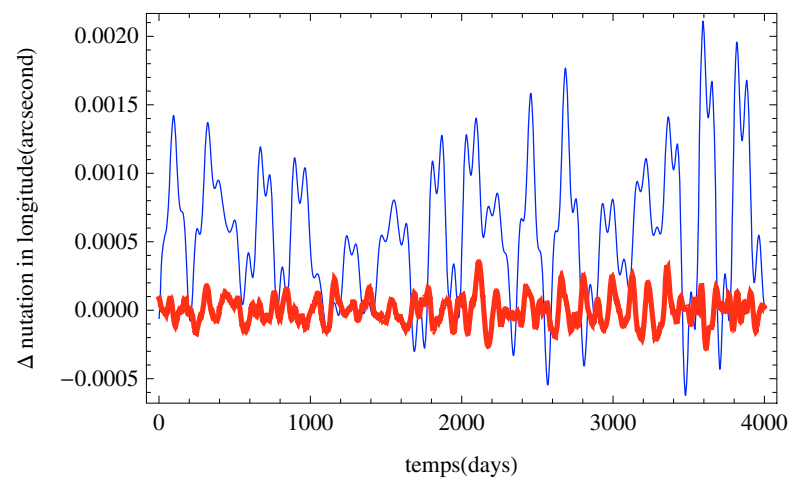

Fig. 11. Difference between the nutation in longitude of the AMA and the numerical integration for $4000 \mathrm{~d}$ time span, from J2000.0. The curve at bottom represents the residual after substracting the sinusoidal terms of the Table 14.

the opposite, when comparing them with the tables of nutation of the Earth taken from Souchay \& Kinoshita (1996), similar periods appear, which correspond to the combination of planetary longitudes. This is a clear confirmation that the differences between our analytical computation and our numerical integration in Figs. 10 and 11, are essentially due to the indirect planetary effects, negligible at first order and not taken previously into account by Cottereau \& Souchay (2009). Tables 13 and 14 also present, when they have been clearly identified, the combination of the planetary longitudes corresponding to the detected sinusoids. We also note the corresponding amplitudes of the Earth's nutation due to the indirect effect of Venus where available. We can thus point out the similitude and reciprocity of the indirect planetary effects of Venus on the Earth rotation, and of the indirect planetary effects of the Earth on the rotation of Venus.

\section{Conclusion and prospects}

We achieved the accurate study of the rotation of Venus for a rigid model and on a short time scale begun by Cottereau \& Souchay (2009) by applying analytical formalisms already used for the rigid Earth (Kinoshita 1972, 1977). The differences between the rotational characteristics of Venus and our planet, due to the slow rotation of Venus and its small obliquity, have been highlighted.

Firstly we precisely determined the polhody, i.e. the torquefree rotational motion for a rigid Venus. We adopted the theory used by Kinoshita $(1972,1992)$ and gave the parametrization and the equations of motion to solve the motion. We showed
Table 13. Rigid Venus nutation coefficient from the indirect planetary contribution in longitude.

\begin{tabular}{crrrrr}
\hline \hline Planetary effects & Period & $\begin{array}{r}\text { Amplitude } \\
\text { sin }\end{array}$ & $\begin{array}{r}\text { Amplitude } \\
\text { cos }\end{array}$ & $\begin{array}{r}\text { SK } \\
\text { sin }\end{array}$ & $\begin{array}{r}\text { SK } \\
\text { cos }\end{array}$ \\
& yr & 0.01 mas & 0.01 mas & 0.01 mas & 0.01 mas \\
\hline 2V-2E & 0.80 & 31.5 & -9.6 & -9.6 & 0.0 \\
2V-3M & 0.60 & 19.3 & 4.0 & $/$ & $/$ \\
3V-3E & 0.53 & -2.0 & 13.4 & 0.5 & -0.2 \\
V-J & 0.64 & 15.1 & -26.2 & $/$ & $/$ \\
V-E & 1.60 & -2.6 & -15.3 & 6.6 & 0.0 \\
& 4.11 & -13.3 & -38.4 & $/$ & $/$ \\
2V+2M+2J & 0.22 & 6.2 & 12.7 & $/$ & $/$ \\
& 9.31 & -274.5 & 421.0 & $/$ & $/$ \\
& 0.20 & -1.5 & -2.3 & $/$ & $/$ \\
2V-2Me & 0.70 & -21.0 & -6.5 & $/$ & $/$ \\
2V+J+M & 0.19 & 8.5 & -7.1 & $/$ & $/$ \\
\hline
\end{tabular}

Notes. Comparison with the respective value in tables of Souchay \& Kinoshita (1995) when we have the Earth as a indirect planetary contribution.

Table 14. Rigid Venus nutation coefficient from the indirect planetary contribution in obliquity.

\begin{tabular}{crrrrr}
\hline \hline \multicolumn{2}{l}{ Planetary effects } & Period Amplitude & Amplitude & SK & SK \\
& & $\begin{array}{c}\text { cos } \\
\text { sin }\end{array}$ & $\begin{array}{c}\text { SK } \\
\text { cos }\end{array}$ \\
\hline 2V+2M+2J & 0.22 & 0.01 mas & 0.01 mas & 0.01 mas & 0.01 mas \\
2S & 14.96 & 0.7 & -0.4 & $/$ & $/$ \\
& 4.06 & -0.3 & 0.2 & $/$ & $/$ \\
2V-2Mer & 0.19 & -0.3 & -0.3 & $/$ & $/$ \\
V-2J+M & 0.50 & -0.3 & 0.0 & $/$ & $/$ \\
\hline
\end{tabular}

Notes. Comparison with the respective value in tables of Souchay \& Kinoshita (1995) when we have the Earth as a indirect planetary contribution.

that the polhody is significantly elliptic, quite different from the Earth, where it can be considered as circular in first approximation. Moreover it is considerably slower. Indeed, the period of the torque-free motion is $525.81 \mathrm{cy}$ for Venus, whereas it is $303 \mathrm{~d}$ for our planet, when considered as rigid.

Then we determined the motion of the third figure axis, which is fundamental from an observational point of view. We calculated the Oppolzer terms due to the gravitational action of the Sun with the equation of motion of Kinoshita (1977) as well as the corresponding development of the disturbing functions. We compared them with the nutation coefficients for the angular momentum axis taken in Cottereau \& Souchay (2009). One of the important results is that these Oppolzer terms depending on the dynamical flattening are of the same order of amplitude as the nutation coefficients themselves, whereas for the Earth (Woolard 1953; Kinoshita 1977) these Oppolzer terms are very small with respect to the nutation coefficients of the angular momentum axis. Moreover we computed the Oppolzer terms depending on the triaxiality, which is not done in Kinoshita (1977) for Earth, which they neglect. For Venus these Oppolzer terms are significant even larger than the corresponding coefficients of nutation of the angular momentum axis.

With our Oppolzer terms we were also able to give the tables of nutation of the third figure axis, from which we computed the nutation for a $4000 \mathrm{~d}$ time span. The comparison with the nutation of the angular momentum axis, calculated from Cottereau \& Souchay (2009), is also given. The nutation of the 
third figure axis is significantly smaller peak to peak than the nutation of the angular momentum axis in longitude, and less important in obliquity. The amplitude of the largest nutation coefficient in longitude of the third figure axis $\left(1.5^{\prime \prime}\right)$ is half that of the angular momentum axis $\left(3^{\prime \prime}\right)$. The amplitude of the nutation in obliquity of the third figure axis is 0 . $^{\prime} 18$ peak to peak, whereas the amplitude of the angular momentum axis is 0.25 . The nutations of the third figure axis, in obliquity and in longitude, are dominated by three sinusoids associated with the arguments $2 L_{\mathrm{s}}, M$ and $2 \Phi$, with respective periods $112.35 \mathrm{~d}, 224.70 \mathrm{~d}$ and $121.51 \mathrm{~d}$. The nutation of the angular momentum axis is dominated by two sinusoids with the argument $2 L_{\mathrm{s}}$ and $2 \Phi$. Our results showed that although the axis of angular momentum and the third figure axis can be considered identical for Earth (Kinoshita 1977), this approximation does not hold for a slowly rotating planet Venus.

We validated our analytical results down to a relative accuracy of $10^{-5}$ with a numerical integration. Moreover, we confirmed by using results in Souchay \& Kinoshita (1996) for the nutation of a rigid Earth that the differences between our analytical computation and our numerical integration are essentially due to the indirect planetary effects, which was not taken into account by Cottereau \& Souchay (2009). This study is fundamental to understand the behaviour of Venus' rotation in a very accurate and exhaustive way for short time scales and will be a starting point for another similar study including non-rigid effects (elasticity, atmospheric forcing etc.).

\section{Appendix}

\subsection{Development for a small value of the triaxiality (Kinoshita 1972)}

We note $\tilde{b}=\sqrt{\frac{G}{\tilde{L}}}$.

$$
\begin{aligned}
l= & \tilde{l}-\left(\frac{1}{4}(\tilde{b}+1) e \sin 2 \tilde{l}\right. \\
& +\left(\frac{1}{64}\left(\tilde{b}^{2}+6 \tilde{b}+1\right) e^{2} \sin 4 \tilde{l}+O\left(e^{3}\right),\right.
\end{aligned}
$$

$$
\begin{aligned}
g= & \tilde{g}+\sqrt{\tilde{b}}\left[\frac{1}{2} e \sin 2 \tilde{l}\right. \\
& \left.-\frac{1}{16}(\tilde{b}+1) e^{2} \sin 4 \tilde{l}\right]+O\left(e^{3}\right)
\end{aligned}
$$

$$
\begin{aligned}
J= & \tilde{J}+\frac{1}{16}(2 \tilde{b}+1) e^{2} \tan \tilde{J} \\
& +\tan \tilde{J}\left(\frac{1}{2} e \cos 2 \tilde{l}-\frac{1}{16} \tilde{b} e^{2} \cos 4 \tilde{l}\right)+O\left(e^{3}\right),
\end{aligned}
$$

with

$$
\begin{aligned}
& \tilde{J}=j+\frac{1}{2} e \tan j+e^{2} \tan j\left(\frac{1}{8}+\frac{3}{16} \tan ^{2} j\right)+O\left(e^{3}\right) \\
& \tilde{l}=\tilde{n_{l}} \times t \quad \text { with } \quad \tilde{n}_{l}=\frac{G}{D} \cos \tilde{J}\left[1-\frac{1}{8}\left(\tilde{b}^{2}+3\right) e^{2}\right]+O\left(e^{4}\right) \\
& \tilde{g}=\tilde{n_{g}} \times t \quad \text { with } \\
& \tilde{n_{g}}=\frac{1}{2}\left(\frac{1}{A}+\frac{1}{B}\right) G+\frac{G}{4 D}(\tilde{b}+1) e^{2}+O\left(e^{4}\right) .
\end{aligned}
$$

\subsection{Development for a small value of the angle $j$ (Kinoshita 1972)}

The polar angles $l$ and $J$ leading to the determination of the free rotational motion are given by

$$
\begin{aligned}
& l= l^{*}-\frac{1}{4} e \sqrt{\frac{1+e}{1-e}} j^{2} \frac{\sin 2 \tilde{l}}{1+e \cos 2 \tilde{l}}+O\left(j^{4}\right) \\
& g=\tilde{g}+\frac{G}{D n_{\tilde{l}}}\left(-\sqrt{1-e^{2}}\left(l^{*}-\tilde{l}\right)\right. \\
&\left.+\frac{1}{4}(1+e) j^{2}\left[\frac{e \sin 2 \tilde{l}}{1+e \cos 2 \tilde{l}}+\frac{2}{\sqrt{1-e^{2}}}\left(l^{*}-\tilde{l}\right)\right]\right)+O\left(j^{4}\right) \\
& J=j \sqrt{1+\frac{2 e}{1-e} \cos ^{2} \tilde{l}}+O\left(j^{3}\right)
\end{aligned}
$$

with

$$
\begin{aligned}
\tan l^{*} & =\sqrt{\frac{1-e}{1+e}} \tan \tilde{l} \\
n_{\tilde{l}}= & \frac{G}{D} \sqrt{\left(1-e^{2}\right)}\left[1-\frac{1}{2(1-e)} j^{2}\right]+O\left(j^{4}\right) \\
n_{\tilde{g}}= & \frac{1}{2}\left(\frac{1}{A}+\frac{1}{B}\right) G+\frac{G}{D}\left(1-\sqrt{1-e^{2}}\right) \\
& \times\left[1+\frac{1}{2} \sqrt{\frac{1+e}{1-e} j^{2}}\right]+O\left(j^{4}\right) .
\end{aligned}
$$

\section{References}

Andoyer, H. 1923 (Paris: Gauthier-Villars et cie)

Byrd, P. F., \& Friedman, M. 1954, Mitteilungen der Astronomischen Gesellschaft Hamburg, 5, 99

Carpenter, R. L. 1964, AJ, 69, 2

Chazy, J. 1953, Mécanique céleste. Équations canoniques et variations des constantes (Paris, Pr. Universitaires de France)

Correia, A. C. M., \& Laskar, J. 2001, Nature, 411, 767

Correia, A. C. M., \& Laskar, J. 2003, Icarus, 163, 24

Cottereau, L., \& Souchay, J. 2009, A\&A, 507, 1635

Deprit, A. 1967, Am. J. Phys., 35, 424

Dobrovolskis, A. R. 1980, Icarus, 41, 18

Goldreich, P., \& Peale, S. J. 1970, AJ, 75, 273

Goldstein, R. M. 1964, AJ, 69, 12

Goldstein, H. 1980, Class. Mech. (Addison-Wesley Publishing Company)

Habibullin, S. T. 1995, Earth Moon and Planets, 71, 43

Hori, G. 1966, PASJ, 18, 287

Kinoshita, H. 1972, PASJ, 24, 423

Kinoshita, H. 1977, Celest. Mech., 15, 277

Kinoshita, H. 1992, Celest. Mech. Dyn. Astron., 53, 365

Kozlovskaya, S. V. 1966, AZh, 43, 1081

Lago, B., \& Cazenave, A. 1979, Moon and Planets, 21, 127

Simon, J. L., Bretagnon, P., Chapront, J., et al. 1994, A\&A, 282, 663

Souchay, J., \& Kinoshita, H. 1996, A\&A, 312, 1017

Standish, E. M. 1998, A\&A, 336, 381

Yoder, C. F. 1995, Icarus, 117, 250

Woolard, E. W. 1953, Astronomical papers prepared for the use of the American ephemeris and nautical almanac (Washington, US: Govt. Print. Off.), 15, 1 